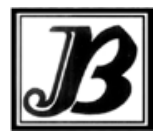

J. Bio-Sci. 28: 21-32, 2020

ISSN 1023-8654 http://www.banglajol.info/index.php/JBS/index DOI: https://doi.org/10.3329/jbs.v28i0.44707

\title{
IN VITRO SCREENING FOR SALT STRESS TOLERANCE OF NATIVE AND EXOTIC POTATO GENOTYPES BY MORPHOLOGICAL AND PHYSIOLOGICAL PARAMETERS
}

\author{
M Harun-Or-Rashid, SMS Islam* and MA Bari \\ Plant Biotechnology and Genetic Engineering Lab., Institute of Biological Sciences, University of Rajshahi, \\ Rajshahi-6205, Bangladesh
}

\begin{abstract}
This study was performed on in vitro bioassay screening for salt tolerance of ten native and six exotic potato genotypes in Bangladesh. Single node was used to evaluate salinity tolerance especially on biomass production. Five different concentrations of $\mathrm{NaCl}(0=$ control, 100, 150, 200 and $250 \mathrm{mM})$ were used in addition to MS medium and evaluated salt tolerant and sensitive genotype by various morphological and physiological parameters e.g. shoot and root length and its thickness, number of leaves and roots, fresh and dry weight of whole plant and water contents. The ANOVA, DMRT and correlation coefficient were found highly significant at $p<0.01$ among the genotypes. With salt stress condition highly positive correlation, co-efficient were found between stem length and internodal distance, leaves number, roots number, root length, dry weight of whole plant and fresh weight of whole plant. A dendrogram based on relative values of 10 morphological and physiological parameters of growth under salt conditions were led to clustering into four distinct group's i.e. tolerant, moderately tolerant, sensitive and very sensitive. On the basis of stress tolerance trait indices (STTIs), Arun (92.78) and Ausha (80.27) showed as a highest salt tolerant, Jamalu (56.33) and Chollisha (57.03) showed the most salt sensitive potato cultivars. From this finding it may be concluded that in vitro screening with bioassay are relatively simple, rapid and convenient and these methods can be used for further advance biotechnological research on potato improvement.
\end{abstract}

Key words: Dendrogram, In vitro, Salt stress, Solanum tuberosum, STTIs

\section{Introduction}

In terms of human consumption potato is the third most important food crop in the world after rice and wheat. More than a billion of the people worldwide eat potato reported by International Potato Center (2016). Annual production of potato is around 385 million tons and yield is 200,511 mt/ha (FAO 2016). Developing countries produced more than half of the total world potato production (Scott 2011). Bangladesh has a high agroecological possibility of growing potato but the average yield is $20.44 \mathrm{mt} / \mathrm{ha}$ (BBS 2018), which is very low in comparison with many other countries due to various biotic and abiotic stress factors (FAO 2016). Plants are sessile and sensitive beings that meet a different environmental stresses throughout the life cycle and its growth and productivity are negatively impressed by various environmental factors (Khan and Singh 2008, Tuteja and Sopory 2008, Yadav et al. 2012). Rengasamy (2006) reported that several parts of the world have been affected by environmental stresses like salt, drought, cold and UV, which hampers crop cultivation and yield. The world population is estimated to attain near 10 billion by 2050, which will find serious food deficits. So, tolerant crops should be developed specially for stress prone area to feed the raising world population (Gill and Tuteja 2010).

*Author for correspondence: shahin_ibsc@ru.ac.bd 
Salinity is an important environmental stress factor that usually becomes critical constraint for crop production (Harun-Or-Rashid et al. 2017, Peethambaran et al. 2018). Moreover, increasing salinization of cultivable land is predicted to have devastating global effects, resulting in $30 \%$ land loss within next 25 years and up to $50 \%$ by the middle of $21^{\text {st }}$ century (Wang et al. 2003). The United Nations Environment Program estimates that approximately $20 \%$ of agricultural land and $50 \%$ of crop land in the world is salt-stressed (Yokoi et al. 2002). Bangladesh has a coastal area of 2.5 million hectares. About $20 \%$ of the net arable land of Bangladesh coastal region is affected by different degrees of salinity (Khanom and Salehin 2012). Agricultural land use in these areas is very poor. Potato is considered as moderately salt sensitive in comparison with other crops since potato tuber yield could be adversely affected at field salinity EC levels of 2.0-3.0 dSm-1 (approx. 22-33 mM NaCl) (Maas and Hoffman 1977).

Genetic variation in salt tolerance within and among plant species can be utilized to screen and select breeding candidates (Epstein and Rains 1987). Conventional field trials are not only time consuming and labour intensive, but also difficult to replicate since the whole plant response to salinity stress is complex and varies with environmental conditions (e.g. season, light, climate, and soil type) (Flowers and Yeo 1989, Siddique et al. 2014). The most effective method to cope with soil salinity problems is through the selection and use of salt tolerant crop plants (Ashraf and Wu 1994). In consideration of the environmental variations in conventional field trials, in vitro conditions can provide faster and more precise evaluations of plant growth under saline and drought stress (Banu et al. 2014). Rahman et al. (2008) observed that salinity effect of three Bangladeshi potato cultivars by using single node cutting. In vitro evaluations of $\mathrm{NaCl}$ or mixed salt stress effects on potato genotypes were proposed as alternatives to the costly, labour intensive and sometimes problematic field based traits (Albiski et al. 2012). Salinity stress of potato has been reported by single-node cuttings (Naik and Widholm 1993), five-node cuttings (Morpurgo 1991), root tip segment or suspension cultures were conducted by Naik and Widholm (1993) and they determined that the relative tolerance of cultivars based on a multivariate analysis of the relative means of six growth parameters of in vitro plantlets over a range of salinity levels. So far there is no report on in vitro salt tolerance screening of Bangladeshi potato genotypes. The main objective of this study was to assess the potential for in vitro screening of ten native and six exotic potato cultivars of Bangladesh for salt tolerance efficiency.

\section{Materials and Methods}

\section{Plant materials and culture conditions}

For evaluating the response in salt stress, ten native potato lines viz. Ausha, Chollisha, Dohazari, Jamalu, Lalpakri, Patnai, Sadaguti, Sheelbilati, Sindurkouta and Surjamukhi and six exotic Potato viz. Arun, Asterix, Cardinal, Courage, Diamant and Granola were considered in this study. As primary explants, developed sprouts were measured 1.0 to $2.0 \mathrm{~cm}$ in length and remove them from the tubers and washed with distilled water followed by plunging in $70 \%$ alcohol for 30 seconds. Then immediately washed with distilled water and subsequently sterilization procedure were done in the laminar air flow cabinet with $0.1 \%$ aqueous solution of $\mathrm{HgCl}_{2}$ for 4-5 minutes. Surface sterilized sprouts were washed 4-5 times with sterilized distilled water and then transferred into $15 \mathrm{ml}$ of MS medium supplemented with 3\% (w/v) sucrose (Merck, Germany) and 0.8\% agar. Then cultures were maintained under a 16/8 $\mathrm{h}$ light/dark photoperiod with 2500 lux light intensity at $25 \pm 1^{\circ} \mathrm{C}$. In order to assess salt tolerance, single nodes derived from sprout culture were transferred into MS (Murashige and Skoog 1962) medium containing five concentrations $(0=$ control, 100, 150, 200 and 250 $\mathrm{mM})(\mathrm{w} / \mathrm{v})$ of $\mathrm{NaCl}$. In this case, four replicates were considered and each treatment consists of 15 explants. Effect of $\mathrm{NaCl}$ were evaluated on following parameters - i) stem length (SL), ii) stem thickness (ST), iii) leaf number (LN), iv) internodal distance (IND), v) root number (RN), vi) root length (RL), vii) root thickness (RT), 
viii) fresh weight of whole plant (FWWP), ix) dry weight of whole plants (DWWP) (oven-dried at $70^{\circ} \mathrm{C}$ for 24 h) and x) plant water content (PWC).

\section{Measurement of various organs}

Six-week old in vitro grown plants from each cultivar were harvested then washed in sterilized water and morphological data were recorded. All plant organs were measured with the help of software ImageJ (public domain Java image processing program) and analyzed by the statistical software SPSS ver. 20.0. Water content (\%) of whole plant and Stress Tolerance Traits Index (STTI) was estimated according to following formula.

\section{Water content (\%) of whole plant}

After measuring fresh weight of whole plant and dry weight, plant water content was estimated by the following formula:

Plant water content $(\%)=\frac{\text { Fresh weight of whole plant }- \text { Dry weight of whole plant }}{\text { Dry weight of whole plant }} \times 100$

\section{Stress tolerance trait index (STTI)}

Major abiotic stress (salt) tolerance index (STI) was estimated as average of STTIs (Shahzad et al. 2012).

STTI $=\frac{\text { Value of trait under stress condition }}{\text { Value of trait under no stress }} \times 100$

Rank

Surviving genotypes at $150 \mathrm{mM}$ salt stress were ranked on the basis of stress tolerance index (STIs).

\section{Experimental design and statistical analysis}

The experiment was designed as completely randomized design with four replications and each replication consist of 15 explants. Data were subjected to ANOVA using the statistical software SPSS ver. 20 and were expressed as Mean \pm standard errors. Pearson correlation analysis was achieved to examine degrees of association between characters, and to perform cluster analysis according to the lines response to salt stress based on the sum of all 10 growth parameters relative values by Ward's method (Ward 1963). A hierarchical dendogram was constructed using PAST (PAleontological STatistics) software.

\section{Results and Discussion}

In vitro screening of potato genotypes for water stress tolerance has been offered as an alternative option to costly, labor-intensive and sometimes problematic field-based screening (Gopal and Iwama 2007, Rahman et al. 2008). Albiski et al. (2012) reported that due to spatial heterogeneity of soil chemical and physical properties and seasonal fluctuations it is difficult to screen a large number of genotypes for salt tolerance in the field. The effect of water or salinity stress on in vitro potato growth has been reported to be similar that observed under field conditions (Zhang and Donnelly 1997, Gopal and Iwama 2007, Khenifi et al. 2011).

In this study potato genotypes showed decreasing in morphological or physiological parameters when $\mathrm{NaCl}$ concentration in the MS medium increased resulting low water potential. Under extreme water stress conditions inducing with $200 \mathrm{mM}, 250 \mathrm{mM} \mathrm{NaCl}$ all genotypes showed less shoots and in some cases did not produced any shoots and leaves. Getting genotypic difference in this case it was very difficult to measure any 
morphological characters. But 100 and $150 \mathrm{mM} \mathrm{NaCl}$, plants produced stems and leaves and no leaf necrosis was found. Differences in morphological characteristics observed only at $150 \mathrm{mM} \mathrm{NaCl}$, while at $100 \mathrm{mM} \mathrm{NaCl}$ plant responses were generally similar to the control. Thus the proposed in vitro system for screening potato against salt stress only a limited level like $150 \mathrm{mM} \mathrm{NaCl}$ can be used for differentiating the genotypes. These results have similarity of findings with single-node cuttings of potato screened for salinity tolerance where use of relatively high level of $\mathrm{NaCl}$ failed to quantify difference among cultivars in vitro (Morpurgo 1991, Khenifi et al. 2011).

\section{Morphological effects on salt stress}

Due to the salt stress factors, stem length (SL) was decreased in all cultivars, ranging from $42.66 \%$ in Surjamukhi to $92.28 \%$ in Sheelbilati (Table 1). Data showed that the significant difference existed in shoot length in different genotypes of potato under salt condition (Table 3). Increases in stem thickness (ST) due to salt were also observed in all cultivars except Asterix (3.66\%) and Courage (13.56\%) (Table 1). It was observed that numbers of leaves were decreased due to salt stress in all cultivars ranging from $6.15 \%$ (Cardinal) to $68.67 \%$ in Sheelbilati (Table 1). Number of leaves was showed significantly differences among the genotypes (Table 3). The high range of variation (3.00-8.88) was found in total number of leaf among the cultivars (Table 3 ). Internodal distances were decreased in all lines because of salt stress. Such reductions ranged were found from $29.69 \%$ in Surjamukhi to $78.57 \%$ in Sindurkouta. The ranges of variation (0.17-0.70

$\mathrm{cm}$ ) were present in the character among the studied genotypes (Table 3).

It was observed that due to salt stress number of roots (13.51\%) was reduced in Patnai and $59.18 \%$ in Courage and the existence of range of variability (1.88-4.00) shown in Table $1 \& 3$. Root length was decreased due to salt stress in all cases; the reduction of root length ranged from $19.82 \%$ for Cardinal to $75.54 \%$ for Sadaguti. Root thickness was increased due to salt from 5.91\% in Asterix to 51.55\% in Arun and reduction of root thickness was observed from $5.54 \%$ in Sindurkouta to $24.52 \%$ in Courage when $150 \mathrm{mM}$ $\mathrm{NaCl}$ was added in medium. Salt caused a decrease in fresh weight of all cultivars, with decreases ranging from $15.73 \%$ in Arun to $83.33 \%$ in Jamalu. In case of dry weight of whole plant, almost all cultivars decreased their weight due to salt $(150 \mathrm{mM} \mathrm{NaCl})$ ranging from $42 \%$ in Ausha to $86.11 \%$ in Chollisha except Arun (Table 1).

It was observed that plant water content (PWC) increases due to salt $(150 \mathrm{mM} \mathrm{NaCl})$ in the cultivars of Ausha (0.77\%), Chollisha (3.69\%), Dohazari (3.43\%), Sadaguti (4.71\%), Sindurkouta (0.60\%), Cardinal (0.58\%), Courage (0.60\%), Diamant (0.39\%) and Granola (2.97\%). But PWC decreased when $150 \mathrm{mM} \mathrm{NaCl}$ added in medium for Jamalu (2.08\%), Lalpakri (1.96\%), Patnai (0.22\%), Sheelbilati (1.89\%), Surjamukhi (0.57\%), Arun (2.35\%) and Asterix (0.95\%) (Table 1).

Analysis of variance on salt effects for different morphological characters viz. SL, ST, LN, IND, RN, RL, RT, FWWP, DWWP and PWC indicated highly significant difference $(p<0.01)$ among the genotypes for all characteristics (Table 2). Mean comparison of the morphological and physiological traits measured under salt stress condition using DMRT showed that cultivar Cardinal had the highest PWC (95.28\%) (Table 3). The highest number of ST, FWWP and DWWP belonged to genotype Arun and highest amount of SL and IND exhibited to genotype Courage. Genotype Ausha, Patnai, Asterix and Diamant had the highest LN, RN, RL and RT, respectively. The result of morphological and physiological traits obviously revealed that culture response was greatly influenced by the potato genotypes. 
Table 1. Effect of salt (150 $\mathrm{mM} \mathrm{NaCl})$ stress factors on different morphological and physiological parameters of different potato genotypes.

\begin{tabular}{|c|c|c|c|c|c|c|c|c|c|c|c|}
\hline \multirow{2}{*}{\multicolumn{2}{|c|}{ Genotypes }} & \multicolumn{2}{|c|}{$\mathrm{SL}(\mathrm{cm})(\mathrm{M} \pm \mathrm{SE})$} & \multicolumn{2}{|c|}{$\mathrm{ST}(\mathrm{mm})(\mathrm{M} \pm \mathrm{SE})$} & \multicolumn{2}{|c|}{$\mathrm{LN}(\mathrm{M} \pm \mathrm{SE})$} & \multicolumn{2}{|c|}{ IND (mm) $(\mathrm{M} \pm \mathrm{SE})$} & \multicolumn{2}{|c|}{$\mathrm{RN}(\mathrm{M} \pm \mathrm{SE})$} \\
\hline & & Cont. & $\begin{array}{c}\text { Salt } \\
(\% \downarrow \uparrow)\end{array}$ & Cont. & $\begin{array}{c}\text { Salt } \\
(\% \downarrow \uparrow)\end{array}$ & Cont. & $\begin{array}{c}\text { Salt } \\
(\% \downarrow \uparrow)\end{array}$ & Cont. & $\begin{array}{c}\text { Salt } \\
(\% \downarrow \uparrow)\end{array}$ & Cont. & $\begin{array}{c}\text { Salt } \\
(\% \downarrow \uparrow)\end{array}$ \\
\hline \multirow{7}{*}{ 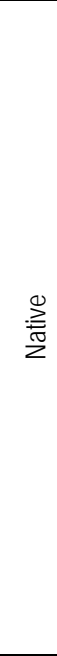 } & Ausha & $7.00 \pm 0.29$ & $\begin{array}{c}3.98 \pm 0.19 \\
(43.19 \downarrow)\end{array}$ & $0.70 \pm 0.03$ & $\begin{array}{c}1.24 \pm 0.06 \\
(76.96 \uparrow)\end{array}$ & $9.87 \pm 0.44$ & $\begin{array}{c}8.88 \pm 0.44 \\
(10.12 \downarrow)\end{array}$ & $0.72 \pm 0.04$ & $\begin{array}{c}0.45 \pm 0.02 \\
(37.34 \downarrow)\end{array}$ & $5.75 \pm 0.25$ & $\begin{array}{c}2.88 \pm 0.15 \\
(50.02 \downarrow)\end{array}$ \\
\hline & Chollisha & $6.61 \pm 0.24$ & $\begin{array}{c}0.88 \pm 0.04 \\
(86.62 \downarrow)\end{array}$ & $0.58 \pm 0.03$ & $\begin{array}{c}0.96 \pm 0.05 \\
(64.37 \uparrow)\end{array}$ & $8.75 \pm 0.37$ & $\begin{array}{c}3.00 \pm 0.16 \\
(65.73 \downarrow)\end{array}$ & $0.76 \pm 0.04$ & $\begin{array}{c}0.30 \pm 0.01 \\
(61.10 \downarrow)\end{array}$ & $5.00 \pm 0.24$ & $\begin{array}{c}2.75 \pm 0.14 \\
(45.01 \downarrow)\end{array}$ \\
\hline & Dohazari & $8.24 \pm 0.26$ & $\begin{array}{c}0.66 \pm 0.03 \\
(92.05 \downarrow)\end{array}$ & $0.69 \pm 0.03$ & $\begin{array}{c}1.23 \pm 0.07 \\
(78.84 \uparrow)\end{array}$ & $11.75 \pm 0.52$ & $\begin{array}{c}3.75 \pm 0.19 \\
(68.10 \downarrow)\end{array}$ & $0.71 \pm 0.03$ & $\begin{array}{c}0.18 \pm 0.01 \\
(75.32 \downarrow)\end{array}$ & $3.63 \pm 0.15$ & $\begin{array}{c}2.68 \pm 0.13 \\
(26.23 \downarrow)\end{array}$ \\
\hline & Lalpakri & $7.61 \pm 0.35$ & $\begin{array}{c}0.91 \pm 0.05 \\
(88.08 \downarrow)\end{array}$ & $0.73 \pm 0.03$ & $\begin{array}{c}0.94 \pm 0.05 \\
(30.03 \uparrow)\end{array}$ & $10.12 \pm 0.49$ & $\begin{array}{c}4.88 \pm 0.24 \\
(51.85 \downarrow)\end{array}$ & $0.76 \pm 0.03$ & $\begin{array}{c}0.19 \pm 0.01 \\
(75.36 \downarrow)\end{array}$ & $4.50 \pm 0.19$ & $\begin{array}{c}2.62 \pm 0.17 \\
(41.78 \downarrow)\end{array}$ \\
\hline & Patnai & $10.48 \pm 0.47$ & $\begin{array}{c}2.96 \pm 0.14 \\
(71.77 \downarrow)\end{array}$ & $0.66 \pm 0.03$ & $\begin{array}{c}0.88 \pm 0.05 \\
(31.98 \uparrow)\end{array}$ & $8.38 \pm 0.36$ & $\begin{array}{c}7.38 \pm 0.37 \\
(11.95 \downarrow)\end{array}$ & $1.27 \pm 0.05$ & $\begin{array}{c}0.40 \pm 0.02 \\
(68.09 \downarrow)\end{array}$ & $4.63 \pm 0.20$ & $\begin{array}{c}4.00 \pm 0.20 \\
(13.51 \downarrow)\end{array}$ \\
\hline & Sadaguti & $9.26 \pm 0.39$ & $\begin{array}{c}1.01 \pm 0.05 \\
(89.12 \downarrow)\end{array}$ & $0.77 \pm 0.04$ & $\begin{array}{c}1.46 \pm 0.07 \\
(90.25 \uparrow)\end{array}$ & $14.25 \pm 0.56$ & $\begin{array}{c}5.00 \pm 0.25 \\
(64.92 \downarrow)\end{array}$ & $0.65 \pm 0.03$ & $\begin{array}{c}0.20 \pm 0.01 \\
(68.42 \downarrow)\end{array}$ & $4.58 \pm 0.20$ & $\begin{array}{c}3.25 \pm 0.16 \\
(28.96 \downarrow)\end{array}$ \\
\hline & Sheelbilati & $7.09 \pm 0.32$ & $\begin{array}{c}0.55 \pm 0.03 \\
(92.28 \downarrow)\end{array}$ & $0.55 \pm 0.02$ & $\begin{array}{c}1.07 \pm 0.06 \\
(93.13 \uparrow)\end{array}$ & $10.38 \pm 0.39$ & $\begin{array}{c}3.25 \pm 0.18 \\
(68.67 \downarrow)\end{array}$ & $0.69 \pm 0.03$ & $\begin{array}{c}0.17 \pm 0.01 \\
(75.40 \downarrow)\end{array}$ & $3.63 \pm 0.13$ & $\begin{array}{c}2.13 \pm 0.11 \\
(41.38 \downarrow)\end{array}$ \\
\hline \multirow{6}{*}{$\begin{array}{l}.0 \\
\text { 离 }\end{array}$} & Arun & $7.69 \pm 0.38$ & $\begin{array}{c}3.88 \pm 0.19 \\
(49.60 \downarrow)\end{array}$ & $1.48 \pm 0.05$ & $\begin{array}{c}2.39 \pm 0.12 \\
(62.20 \uparrow)\end{array}$ & $10.00 \pm 0.50$ & $\begin{array}{c}7.75 \pm 0.39 \\
(22.50 \downarrow)\end{array}$ & $0.77 \pm 0.04$ & $\begin{array}{c}0.50 \pm 0.03 \\
(34.84 \downarrow)\end{array}$ & $5.75 \pm 0.22$ & $\begin{array}{c}3.23 \pm 0.16 \\
(43.91 \downarrow)\end{array}$ \\
\hline & Asterix & $9.60 \pm 0.42$ & $\begin{array}{c}3.62 \pm 0.18 \\
(62.34 \downarrow)\end{array}$ & $1.12 \pm 0.04$ & $\begin{array}{c}1.08 \pm 0.06 \\
(3.66 \downarrow)\end{array}$ & $8.63 \pm 0.35$ & $\begin{array}{c}5.50 \pm 0.29 \\
(36.23 \downarrow)\end{array}$ & $1.16 \pm 0.06$ & $\begin{array}{c}0.66 \pm 0.03 \\
(42.84 \downarrow)\end{array}$ & $4.75 \pm 0.22$ & $\begin{array}{c}3.50 \pm 0.18 \\
(26.24 \downarrow)\end{array}$ \\
\hline & Cardinal & $8.81 \pm 0.36$ & $\begin{array}{c}2.61 \pm 0.13 \\
(70.40 \downarrow)\end{array}$ & $1.27 \pm 0.04$ & $\begin{array}{c}1.45 \pm 0.07 \\
(13.75 \uparrow)\end{array}$ & $8.13 \pm 0.39$ & $\begin{array}{c}7.63 \pm 0.38 \\
(6.15 \downarrow)\end{array}$ & $0.61 \pm 0.03$ & $\begin{array}{c}0.36 \pm 0.01 \\
(71.32 \downarrow)\end{array}$ & $7.75 \pm 0.24$ & $\begin{array}{c}3.32 \pm 0.19 \\
(57.16 \downarrow)\end{array}$ \\
\hline & Courage & $10.54 \pm 0.46$ & $\begin{array}{c}5.48 \pm 0.28 \\
(48.02 \downarrow)\end{array}$ & $1.11 \pm 0.05$ & $\begin{array}{c}0.96 \pm 0.05 \\
(13.56 \downarrow)\end{array}$ & $10.50 \pm 0.51$ & $\begin{array}{c}8.00 \pm 0.41 \\
(23.82 \downarrow)\end{array}$ & $1.03 \pm 0.04$ & $\begin{array}{c}0.70 \pm 0.03 \\
(32.30 \downarrow)\end{array}$ & $6.13 \pm 0.20$ & $\begin{array}{c}2.50 \pm 0.14 \\
(59.18 \downarrow)\end{array}$ \\
\hline & Diamant & $7.25 \pm 0.30$ & $\begin{array}{c}1.83 \pm 0.09 \\
(74.77 \downarrow)\end{array}$ & $1.13 \pm 0.05$ & $\begin{array}{c}1.28 \pm 0.06 \\
(14.04 \uparrow)\end{array}$ & $10.38 \pm 0.41$ & $\begin{array}{c}6.50 \pm 0.33 \\
(37.35 \downarrow)\end{array}$ & $0.73 \pm 0.03$ & $\begin{array}{c}0.29 \pm 0.02 \\
(60.85 \downarrow)\end{array}$ & $4.74 \pm 0.23$ & $\begin{array}{c}2.26 \pm 0.16 \\
(52.26 \downarrow)\end{array}$ \\
\hline & Granola & $9.44 \pm 0.30$ & $\begin{array}{c}1.80 \pm 0.10 \\
(80.89 \downarrow)\end{array}$ & $0.93 \pm 0.04$ & $\begin{array}{c}1.01 \pm 0.05 \\
(8.57 \uparrow) \\
\end{array}$ & $12.13 \pm 0.47$ & $\begin{array}{c}7.55 \pm 0.40 \\
(37.73 \downarrow) \\
\end{array}$ & $0.79 \pm 0.03$ & $\begin{array}{c}0.24 \pm 0.01 \\
(69.28 \downarrow)\end{array}$ & $5.50 \pm 0.19$ & $\begin{array}{c}2.75 \pm 0.15 \\
(50.00 \downarrow) \\
\end{array}$ \\
\hline
\end{tabular}

Cont. = control, SL = stem length, ST = stem thickness, LN = leaves number, IND = internodal distance, RN = roots number, $\downarrow=$ Decrease and $\uparrow=$ Increase. 
Contd. Table 1

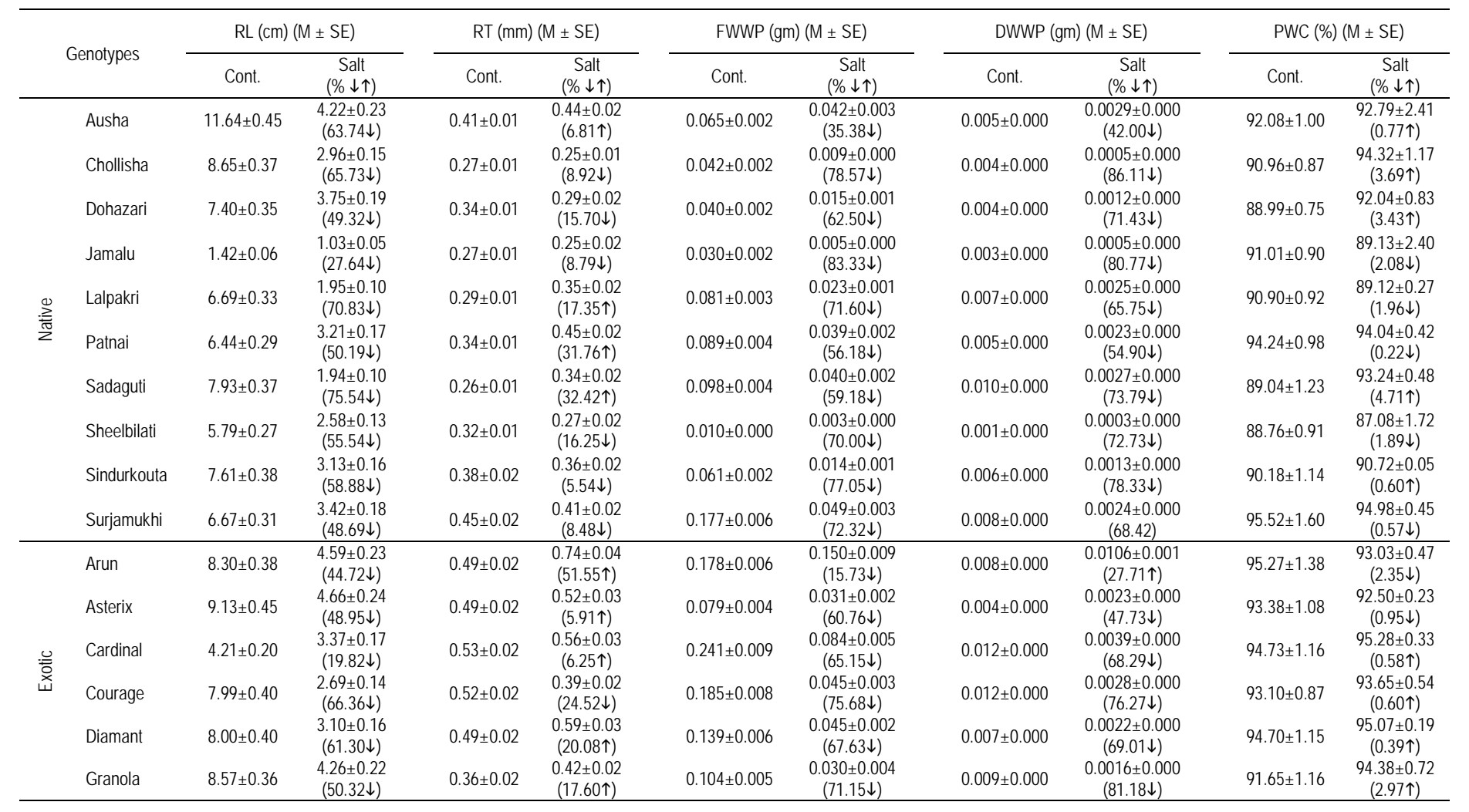

Cont. = control, $\mathrm{RL}=$ root length, $\mathrm{RT}=$ root thickness, FWWP = fresh weight of whole plant, $\mathrm{DWWP}=$ dry weight of whole plant and PWC = plant water content, $\downarrow=$ Decrease and $\uparrow=$ Increase. 
Table 2. Analysis of variance for salt stress effects on various characteristics of potato

\begin{tabular}{|c|c|c|c|c|c|c|c|c|c|c|c|}
\hline \multirow{2}{*}{ SOV } & \multirow{2}{*}{ df } & \multicolumn{10}{|c|}{ MS } \\
\hline & & SL & ST & LN & IND & RN & $\mathrm{RL}$ & RT & FWWP & DWWP & PWC \\
\hline $\begin{array}{l}\text { Genotype } \\
\text { in salt }\end{array}$ & 15 & $143.04^{* *}$ & $9.03^{* *}$ & $232.03^{\text {t* }}$ & $1.78^{* *}$ & $17.71^{* *}$ & $60.76^{* *}$ & $1.1^{\text {*t }}$ & $0.078^{* *}$ & $0.00^{* *}$ & $349.12^{* *}$ \\
\hline Error & 48 & 3.436 & 0.781 & 19.938 & 0.069 & 4.662 & 5.525 & 0.11 & 0.002 & 0.00 & 221.88 \\
\hline
\end{tabular}

SOV: source of variance, ${ }^{*}$ significant at $1 \%$ level of probability. $\mathrm{SL}=$ Stem length, $\mathrm{ST}=$ stem thickness, $\mathrm{LN}=$ leaves number, $I N D=$ internodal distance, $\mathrm{RN}=$ roots number, $\mathrm{RL}=$ root length, $\mathrm{RT}=$ root thickness, FWWP $=$ fresh weight of whole plant, DWWP $=$ dry wt of whole plant and PWC $=$ plant water content as foot note.

Table 3. Mean comparison for morphological data under salt stress condition using DMRT

\begin{tabular}{lcccccccccc}
\hline Genotypes & $\mathrm{SL}$ & $\mathrm{ST}$ & $\mathrm{LN}$ & $\mathrm{IND}$ & $\mathrm{RN}$ & $\mathrm{RL}$ & $\mathrm{RT}$ & FWWP & DWWP & PWC \\
\hline Ausha & $3.98 \mathrm{e}$ & $1.24 \mathrm{def}$ & $8.88 \mathrm{f}$ & $0.45 \mathrm{ef}$ & $2.88 \mathrm{defg}$ & $4.22 \mathrm{gh}$ & $0.44 \mathrm{f}$ & $0.04 \mathrm{~h}$ & $0.0029 \mathrm{e}$ & $92.79 \mathrm{~cd}$ \\
Chollisha & $0.88 \mathrm{ab}$ & $0.96 \mathrm{bc}$ & $3.00 \mathrm{a}$ & $0.30 \mathrm{c}$ & $2.75 \mathrm{cdef}$ & $2.96 \mathrm{cde}$ & $0.25 \mathrm{a}$ & $0.01 \mathrm{abc}$ & $0.0005 \mathrm{a}$ & $94.32 \mathrm{~d}$ \\
Dohazari & $0.66 \mathrm{ab}$ & $1.23 \mathrm{def}$ & $3.75 \mathrm{ac}$ & $0.18 \mathrm{a}$ & $2.68 \mathrm{cde}$ & $3.75 \mathrm{fg}$ & $0.29 \mathrm{abc}$ & $0.02 \mathrm{~cd}$ & $0.0012 \mathrm{ab}$ & $92.04 \mathrm{bcd}$ \\
Jamalu & $0.75 \mathrm{a}$ & $0.65 \mathrm{a}$ & $3.50 \mathrm{a}$ & $0.22 \mathrm{ab}$ & $1.88 \mathrm{a}$ & $1.03 \mathrm{a}$ & $0.25 \mathrm{a}$ & $0.01 \mathrm{ab}$ & $0.0005 \mathrm{a}$ & $89.13 \mathrm{ab}$ \\
Lalpakri & $0.91 \mathrm{ab}$ & $0.94 \mathrm{bc}$ & $4.88 \mathrm{~b}$ & $0.19 \mathrm{ab}$ & $2.62 \mathrm{~cd}$ & $1.95 \mathrm{~b}$ & $0.35 \mathrm{~cd}$ & $0.02 \mathrm{fde}$ & $0.0025 \mathrm{de}$ & $89.12 \mathrm{ab}$ \\
Patnai & $2.96 \mathrm{~d}$ & $0.88 \mathrm{~b}$ & $7.38 \mathrm{de}$ & $0.40 \mathrm{de}$ & $4.00 \mathrm{i}$ & $3.21 \mathrm{de}$ & $0.45 \mathrm{f}$ & $0.04 \mathrm{fgh}$ & $0.0023 \mathrm{cde}$ & $94.04 \mathrm{~cd}$ \\
Sadaguti & $1.01 \mathrm{~b}$ & $1.46 \mathrm{~g}$ & $5.00 \mathrm{~b}$ & $0.20 \mathrm{ab}$ & $3.25 \mathrm{fgh}$ & $1.94 \mathrm{~b}$ & $0.34 \mathrm{bcd}$ & $0.04 \mathrm{gh}$ & $0.0027 \mathrm{e}$ & $93.24 \mathrm{~cd}$ \\
Sheelbilati & $0.55 \mathrm{a}$ & $1.07 \mathrm{bcd}$ & $3.25 \mathrm{a}$ & $0.17 \mathrm{a}$ & $2.13 \mathrm{ab}$ & $2.58 \mathrm{c}$ & $0.27 \mathrm{ab}$ & $0.003 \mathrm{a}$ & $0.0003 \mathrm{a}$ & $87.08 \mathrm{a}$ \\
Sindurkouta & $0.96 \mathrm{ab}$ & $1.35 \mathrm{fg}$ & $5.75 \mathrm{bc}$ & $0.17 \mathrm{a}$ & $2.75 \mathrm{cdef}$ & $3.13 \mathrm{de}$ & $0.38 \mathrm{cde}$ & $0.01 \mathrm{bcd}$ & $0.0013 \mathrm{abc}$ & $90.72 \mathrm{bc}$ \\
Surjamukhi & $3.76 \mathrm{e}$ & $1.14 \mathrm{cde}$ & $8.25 \mathrm{ef}$ & $0.46 \mathrm{ef}$ & $3.17 \mathrm{efgh}$ & $3.42 \mathrm{ef}$ & $0.41 \mathrm{def}$ & $0.05 \mathrm{~h}$ & $0.0024 \mathrm{de}$ & $94.98 \mathrm{~d}$ \\
Arun & $3.88 \mathrm{e}$ & $2.39 \mathrm{f}$ & $7.75 \mathrm{e}$ & $0.50 \mathrm{f}$ & $3.23 \mathrm{fgh}$ & $4.59 \mathrm{~h}$ & $0.74 \mathrm{~h}$ & $0.15 \mathrm{j}$ & $0.0106 \mathrm{~g}$ & $93.03 \mathrm{~cd}$ \\
Asterix & $3.62 \mathrm{e}$ & $1.08 \mathrm{bcd}$ & $5.50 \mathrm{~b}$ & $0.66 \mathrm{~g}$ & $3.50 \mathrm{~h}$ & $4.66 \mathrm{~h}$ & $0.52 \mathrm{~g}$ & $0.03 \mathrm{efg}$ & $0.0023 \mathrm{cde}$ & $92.50 \mathrm{bcd}$ \\
Cardinal & $2.61 \mathrm{~d}$ & $1.45 \mathrm{~g}$ & $7.63 \mathrm{e}$ & $0.36 \mathrm{~d}$ & $3.32 \mathrm{gh}$ & $3.37 \mathrm{ef}$ & $0.56 \mathrm{efg}$ & $0.08 \mathrm{i}$ & $0.0039 \mathrm{f}$ & $95.28 \mathrm{~d}$ \\
Courage & $5.48 \mathrm{f}$ & $0.96 \mathrm{bc}$ & $8.00 \mathrm{e}$ & $0.70 \mathrm{~g}$ & $2.50 \mathrm{bcd}$ & $2.69 \mathrm{~cd}$ & $0.39 \mathrm{def}$ & $0.05 \mathrm{~h}$ & $0.0028 \mathrm{e}$ & $93.65 \mathrm{~cd}$ \\
Diamant & $1.83 \mathrm{c}$ & $1.28 \mathrm{efg}$ & $6.50 \mathrm{~cd}$ & $0.29 \mathrm{c}$ & $2.26 \mathrm{abc}$ & $3.10 \mathrm{cde}$ & $0.59 \mathrm{~g}$ & $0.05 \mathrm{~h}$ & $0.0022 \mathrm{cde}$ & $95.07 \mathrm{~d}$ \\
Granola & $1.80 \mathrm{c}$ & $1.01 \mathrm{bc}$ & $7.55 \mathrm{e}$ & $0.24 \mathrm{bc}$ & $2.75 \mathrm{cdef}$ & $4.26 \mathrm{gh}$ & $0.42 \mathrm{ef}$ & $0.03 \mathrm{ef}$ & $0.0016 \mathrm{bcd}$ & $94.38 \mathrm{~d}$ \\
\hline
\end{tabular}

Mean values followed by the same letter are not significantly different according to Duncan's Multiple Range Test.

$\mathrm{SL}=$ stem length, $\mathrm{ST}=$ stem thickness, $\mathrm{LN}=$ leaves number, IND = internodal distance, $\mathrm{RN}=$ roots number, $\mathrm{RL}=$ root length, $\mathrm{RT}=$ root thickness, FWWP = fresh weight of whole plant, DWWP = dry weight of whole plant and PWC = plant water content as foot note. 


\section{Stress Tolerance Trait Index (STTI)}

Plant growth was determined by number of plants, leaves, root and shoot length and fresh and dry weights. It was observed that $150 \mathrm{mM} \mathrm{NaCl}$ stress showed STTI of shoot length ranged from $7.72 \%$ (Sheelbilati) to 57.34\% (Surjamukhi) (Table 4). Maximum (193.82\%) STTI for shoot thickness was recorded in Sindurkouta and minimum (86.44\%) in Courage. Table 4 represents data on salt tolerance trait indices of leaf number ranged from $31.33 \%$ (Sheelbilati) to $93.85 \%$ (Cardinal). Maximum (70.37\%) STTI for internodal distance was gained by Surjamukhi and minimum (21.43\%) by Sindurkouta. Under $\mathrm{NaCl}(150 \mathrm{mM})$ stress root plays a vital role for plant survival and growth in adverse environmental conditions. STTI of root number varied from 40.82\% (Courage) to $86.49 \%$ (Patnai) (Table 4). Generally tolerant cultivars revealed better root growth than susceptible ones. Another important salt tolerance trait index of root length ranged from $24.46 \%$ (Sadaguti) to $80.18 \%$ (Cardinal). Similar to shoot thickness, STTI of root thickness was also increased. It was ranged from $75.48 \%$ (Courage) to $151.55 \%$ (Arun). Fresh and dry weights of whole plants were affected by $150 \mathrm{mM}$ salinity stress. The STTI values of fresh weight of whole plant ranged from $16.67 \%$ (Jamalu) to $84.27 \%$ (Arun). STTI values of dry weight of whole plant varied from 13.89\% (Chollisha) to $127.71 \%$ (Arun). The stress tolerance trait index in salt of plant water content ranged from 97.65\% (Arun) to $103.69 \%$ (Chollisha). According to the stress tolerant indexes (STIS) ranking, studied genotypes are arranged as below:

Arun $>$ Ausha $>$ Patnai $>$ Cardinal $>$ Surjamukhi $>$ Asterix $>$ Sadaguti $>$ Sindurkouta $>$ Dohazari $>$ Diamant $>$ Sheelbilati> Granola $>$ Courage $>$ Lalpakri>Chollisha $>$ Jamalu.

Table 4. STTIs of 10 morphological traits under $150 \mathrm{mM} \mathrm{NaCl}$ stress on sixteen genotypes

\begin{tabular}{lcccccccccccc}
\hline Genotype & SL & ST & LN & IND & RN & RL & RT & FWWP & DWWP & PWC & STI & Rank \\
& $\%$ & $\%$ & $\%$ & $\%$ & $\%$ & $\%$ & $\%$ & $\%$ & $\%$ & $\%$ & $\%$ & \\
\hline Ausha & 56.81 & 176.96 & 89.88 & 62.66 & 49.98 & 36.26 & 106.81 & 64.62 & 58.00 & 100.77 & 80.27 & 2 \\
Chollisha & 13.38 & 164.37 & 34.27 & 38.90 & 54.99 & 34.27 & 91.08 & 21.43 & 13.89 & 103.69 & 57.03 & 15 \\
Dohazari & 7.95 & 178.84 & 31.90 & 24.68 & 73.77 & 50.68 & 84.30 & 37.50 & 28.57 & 103.43 & 62.16 & 9 \\
Jamalu & 13.35 & 114.34 & 33.32 & 39.71 & 65.22 & 72.36 & 91.21 & 16.67 & 19.23 & 97.92 & 56.33 & 16 \\
Lalpakri & 11.92 & 130.03 & 48.15 & 24.64 & 58.22 & 29.17 & 117.35 & 28.40 & 34.25 & 98.04 & 58.02 & 14 \\
Patnai & 28.23 & 131.98 & 88.05 & 31.91 & 86.49 & 49.81 & 131.76 & 43.82 & 45.10 & 99.78 & 73.69 & 3 \\
Sadaguti & 10.88 & 190.25 & 35.08 & 31.58 & 71.04 & 24.46 & 132.42 & 40.82 & 26.21 & 104.71 & 66.74 & 7 \\
Sheelbilati & 7.72 & 193.13 & 31.33 & 24.60 & 58.62 & 44.46 & 83.75 & 30.00 & 27.27 & 98.11 & 59.90 & 11 \\
Sindurkouta & 12.01 & 193.82 & 56.09 & 21.43 & 57.89 & 41.12 & 94.46 & 22.95 & 21.67 & 100.60 & 62.20 & 8 \\
Surjamukhi & 57.34 & 102.15 & 81.47 & 70.37 & 72.43 & 51.31 & 91.52 & 27.68 & 31.58 & 99.43 & 68.53 & 5 \\
Arun & 50.40 & 162.20 & 77.50 & 65.16 & 56.09 & 55.28 & 151.55 & 84.27 & 127.71 & 97.65 & 92.78 & 1 \\
Asterix & 37.66 & 96.34 & 63.77 & 57.16 & 73.76 & 51.05 & 105.91 & 39.24 & 52.27 & 99.05 & 67.62 & 6 \\
Cardinal & 29.60 & 113.75 & 93.85 & 58.68 & 42.84 & 80.18 & 106.25 & 34.85 & 31.71 & 100.58 & 69.23 & 4 \\
Courage & 51.98 & 86.44 & 76.18 & 67.70 & 40.82 & 33.64 & 75.48 & 24.32 & 23.73 & 100.60 & 58.09 & 13 \\
Diamant & 25.23 & 114.04 & 62.65 & 39.15 & 47.74 & 38.70 & 120.08 & 32.37 & 30.99 & 100.39 & 61.14 & 10 \\
Granola & 19.11 & 108.57 & 62.27 & 30.72 & 50.00 & 49.68 & 117.60 & 28.85 & 18.82 & 102.97 & 58.86 & 12 \\
\hline \multicolumn{1}{c}{ Average } & 27.10 & 141.07 & 60.36 & 43.06 & 59.99 & 46.40 & 106.35 & 36.11 & 36.94 & 100.48 & 65.79 & \\
\hline
\end{tabular}

$\mathrm{SL}=$ stem length, $\mathrm{ST}=$ stem thickness, $\mathrm{LN}=$ leaves number, $\mathrm{IND}=$ internodal distance, $\mathrm{RN}=$ roots number, $\mathrm{RL}=$ root length, RT = root thickness, FWWP = fresh weight of whole plant, DWWP $=$ dry weight of whole plant and PWC $=$ plant water content as foot note. 
Correlations co-efficient between morphological parameters due to salt stress conditions were found highly significant for most of the parameters considered under this study (Table 5). For example, shoot length appeared as a function of internodal distance $\left(R^{2}=0.93\right)$, leaf number $\left(R^{2}=0.83\right)$, root number $\left(R^{2}=0.80\right)$, root length $\left(R^{2}=0.78\right)$, fresh weight of whole plant $\left(R^{2}=0.61\right)$ and dry weight of whole plant $\left(R^{2}=0.71\right)$, while leaf number appeared as a function of internodal distance $\left(R^{2}=0.66\right)$, root number $\left(R^{2}=0.59\right)$, root length $\left(R^{2}=0.69\right)$ and dry weight of whole plant $\left(R^{2}=0.63\right)$. Root number appeared as a function of fresh weight of whole plant $\left(R^{2}=0.78\right)$ and dry weight of whole plant $\left(R^{2}=0.79\right)$. Fresh weight of whole plant appeared as a function of dry weight of whole plant $\left(R^{2}=0.92\right)$.

Table 5. Correlation of the morphological parameters for salt stress on 32 soma clones (control and stresses)

\begin{tabular}{cccccccccc}
\hline Chart. & SL & ST & LN & IND & RN & RL & RT & FWWP & DWWP \\
\hline ST & $-0.288^{\text {ns }}$ & & & & & & & & \\
LN & $0.833^{\star \star}$ & $-0.250^{\text {ns }}$ & & & & & & & \\
IND & $0.927^{\star \star}$ & $-0.271^{\text {ns }}$ & $0.658^{\star \star}$ & & & & & & \\
RN & $0.796^{\star \star}$ & $-0.071^{\text {ns }}$ & $0.585^{\star \star}$ & $0.673^{\star \star}$ & & & & & \\
RL & $0.777^{\star \star}$ & $-0.196^{\text {ns }}$ & $0.689^{\star \star}$ & $0.744^{\star \star}$ & $0.725^{\star \star}$ & & & & \\
RT & $0.103^{\text {ns }}$ & $0.724^{\star \star}$ & $0.097^{\text {ns }}$ & $0.148^{\text {ns }}$ & $0.232^{\text {ns }}$ & $0.113^{\text {ns }}$ & & & \\
FWWP & $0.614^{\star \star}$ & $0.377^{\star}$ & $0.479^{\star \star}$ & $0.487^{\star \star}$ & $0.777^{\star \star}$ & $0.443^{\star}$ & $0.558^{\star \star}$ & & \\
DWWP & $0.714^{\star \star}$ & $0.270^{\text {ns }}$ & $0.634^{\star \star}$ & $0.545^{\star \star}$ & $0.788^{\star \star}$ & $0.549^{\star \star}$ & $0.433^{\star}$ & $0.916^{\star \star}$ & \\
PWC & $0.057^{\text {ns }}$ & $0.444^{\star}$ & $-0.003^{\text {ns }}$ & $0.145^{\text {ns }}$ & $0.239^{\text {ns }}$ & $0.029^{\text {ns }}$ & $0.596^{\star \star}$ & $0.480^{\star \star}$ & $0.222^{\text {ns }}$ \\
\hline
\end{tabular}

* and **significant at $5 \%$ and $1 \%$ level of probability respectively, ns: non-significant

$\mathrm{SL}=$ Stem length, $\mathrm{ST}=$ Stem thickness, $\mathrm{LN}=$ Leaves number, IND = Internodal distance, $\mathrm{RN}=$ Roots number, $\mathrm{RL}=$ Root length, RT = Root thickness, FWWP = Fresh weight of whole plant and DWWP = Dry weight of whole plant as foot note.

\section{Cluster analysis}

For salt stress hierarchical dendrogram showed that the 16 potato genotypes were categorized into four main clusters: (1) Cluster-A salt tolerant group contained four genotypes, i.e., Arun, Ausha, Courage and Asterix. (2) Cluster-B moderately tolerant group consisting of five potato genotypes i.e., Granola, Diamant, Cardinal, Surjamukhi and Patnai.(3) Cluster-C salt sensitive group included four genotypes, i.e. Sadaguti, Chollisha, Dohazari and Sindurkouta and (4) Cluster-D very sensitive group consist of three potato genotypes i.e. Lalpakri, Jamalu and Sheelbilati (Fig. 1). It has been reported that Diamant and Cardinal has salt sensitive cultivars (Khrais et al. 1998, Rahnama and Ebrahimzadeh 2005). In this case Cardinal and Diamant showed as a moderately salt tolerant cultivar. Evaluation to salt stress conditions similar types of results found by Aghaei et al. (2008). This difference might be due to the variation in experimental conditions or the range of salt concentrations in the culture medium which has been tested.

So far as we know till a very little work has been done on abiotic stress screening in Bangladeshi indigenous potato genotypes. An indigenous potato cultivar Sheelbilati has been investigated as salt tolerant by Rahman et al. (2008). Khatun et al. $(2010,2011)$ reported that in their experiment that some local varieties namely Sadaguti and Zaubilati have the potentially to perform better in vivo salt stress condition. In this case results 
obtained on 10 indigenous potato cultivars of Bangladesh and among these indigenous cultivars Sheelbilati showed very salt sensitive. This dissimilarity might be due to the different range of salt concentration in the medium which has been examined.

In comparison with other species, potato is moderately salt sensitive (Ochatt et al. 1999, Aghaei et al. 2008). Based on the relative values of shoot length, fresh and dry weight as well as root length, fresh and dry weight Zhang and Donnelly (1997) screened in vitro genotypes of potato for salinity tolerance. Physiological parameters were used by Aghaei et al. (2008), with random amplification of polymorphic DNA screening to confirm the reliability of screening using physiological parameters. Ranalli et al. (1996) showed that differences in canopy temperature between irrigated and stressed treatments can be used for screening for drought tolerance among potato genotypes.

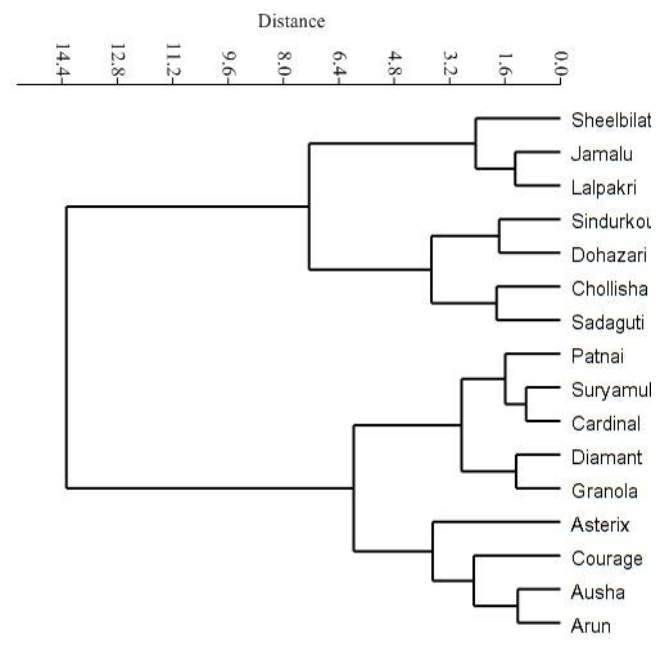

Fig. 1. Dendrogram based on relative values of ten morphological and physiological parameters of growth of potato lines under salt stress. Cluster-A (salt tolerant group): Arun, Ausha, Courage and Asterix. (2) ClusterB (moderately tolerant group): Granola, Diamant, Cardinal, Surjamukhi and Patnai. (3) Cluster-C (salt sensitive group): Sadaguti, Chollisha, Dohazari and Sindurkouta, and (4) Cluster-D (very sensitive group): Lalpakri, Jamalu and Sheelbilati.

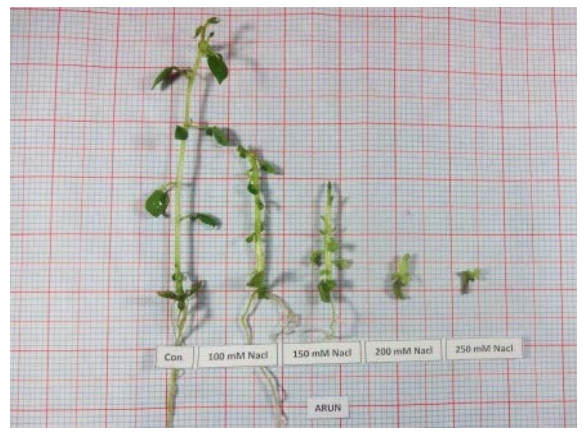

A

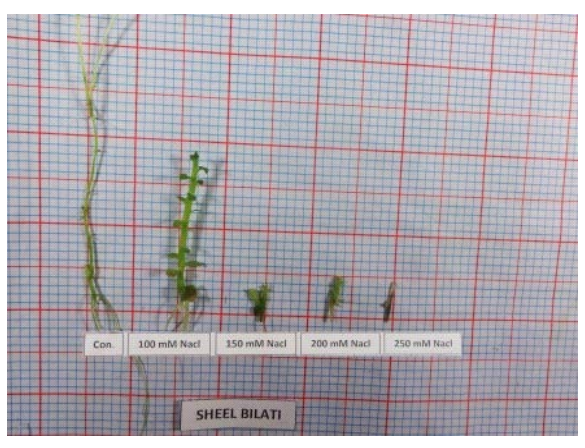

B

Fig. 2. A. Salt tolerant (Arun), and B. Salt sensitive (Sheelbilati) genotype. 


\section{Conclusion}

Screening practice of the potato cultivars suggested that the salt tolerance and sensitivity of some potato cultivars are genotype dependent and maybe not epigenetic adaptation under stress condition. This is also clear from these experiments that in vitro screening with bioassay is relatively simple, rapid, convenient and repeatable. They can be used to substitute for field trials to screen and select salt tolerant potato genotype for further potato improvement in Bangladesh through biotechnological and breeding methods.

\section{Acknowledgements}

The author (MHR) is gratefully acknowledges to the Institute of Biological Sciences, University of Rajshahi, Bangladesh for research facilities and the HEQEP (CP-2485) of University Grants Commission (UGC) of Bangladesh for providing fellowship for this research.

\section{References}

Aghaei K, Ehsanpour AA, Balali G and Mostajeran A (2008). In vitro screening of potato (Solanum tuberosum L.) cultivars for salt tolerance using physiological and RAPD analysis. Am Eur J Agric Env Sci., 3(2): 159-164.

Albiski F, Najla S, Sanoubar R, Alkabani N and Murshed R (2012). In vitro screening of potato lines for drought tolerance. Physiol Mol Biol Plants, 18(4): 315-321.

Ashraf MY and Wu L (1994). Breeding for salinity tolerance in plants. Crit. Rev Plant Sci., 13: 17-42.

Banu MSA, Huda KMK, Shaoo RK, Garg B, Tula S, Islam SMS, Tuteja R and Tuteja N (2014). Pea p68 imparts salinity stress tolerance in rice by scavenging of ROS-mediated $\mathrm{H}_{2} \mathrm{O}_{2}$ and interacts with argonaute. Plant Mol. Biol. Reporter, pp. 1-18.

BBS (2018). Yearbook of Agricultural Statistics-2017, April 2018, Bangladesh Bureau of Statistics. Ministry of Planning. Govt. of the People's Republic of Bangladesh, Dhaka. pp. 574.

CIP (2016). International Potato Center. Potato. Global production. http://cipotato.org/ potato.

Epstein E and Rains DW (1987). Advances in salt tolerance. Plant Soil, 99: 17-29.

FAO (2016). Statistical databases, FAOSTAT, http://faostat3.fao.org/browse/Q/QC/E.

Flowers TJ and Yeo AR (1989). Effect of salinity on plant growth and crop yield. In: Biochemical and physiological mechanisms associated with environmental stress tolerance in plants. Cherry J. (ed.). Springer-Verlag, Berlin, pp. 151-165.

Gill SS and Tuteja N (2010). Polyamines and abiotic stress tolerance in plants. Plant Signaling Behavior 5(1): 26-33.

Gopal J and Iwama K (2007). In vitro screening of potato against water stress mediated through sorbitol and polyethylene glycol. Plant Cell Rep., 26: 693-700.

Harun-Or-Rashid M, Bari MA and Islam SMS (2017). Improvement of cold tolerance efficiency on storage conditions of encapsulated nodal segments of potato using salicylic acid. SKUAST J Res., 19(1): 109-114.

Khan NA and Singh S (2008). Abiotic Stress and Plant Responses. IK International Publishing House Pvt. Ltd., New Delhi.

Khanom S and Salehin M (2012). Salinity constraints to different water uses in coastal area of Bangladesh: A case study. Bangladesh. J Sci Res., 25(1): 33-42.

Khatun MM, Akhond MAY, Molla MMH, Khan ASMMR and Al-Amin M (2010). In vitro screening of potato cultivars for relative salt tolerance. Research Report (2009-2010), Biotechnology Division, Bangladesh Agricultural Research Institute, Gazipur.

Khatun MM, Molla MMH and Akhond MAY (2011). In vitro screening of potato cultivars for relative salt tolerance. Research Report (2010-2011). Biotechnology Division, Bangladesh Agricultural Research Institute, Gazipur. 
Khenifi ML, Boudjeniba M and Kameli A (2011). Effects of salt stress on micropropagation of potato (Solanum tuberosum L.). Af J Biotech., 10(40): 7840-7845.

Khrais T, Leclerc $Y$ and Donnelly D (1998). Relative salinity tolerance of potato cultivars assessed by in vitro screening. Am J Potato Res., 75: 207-210.

Maas EV and Hoffman GJ (1977). Crop salt tolerance-current assessment. J Irrig Drain, 103: 115-134.

Morpurgo R (1991). Correlation between potato clones grown in vivo and in vitro under sodium chloride stress conditions. Plant Breed., 107: 80-82.

Murashige T and Skoog F (1962). A revised medium for rapid growth and bioassays with tobacco tissue cultures. Physiologia Plantarum, 15: 463-497.

Naik PS and Widholm JM (1993). Comparison of tissue culture and whole plant responses to salinity in potato. Plant Cell Tiss Organ Cult., 33: 273-280.

Ochatt SJ, Marconi PL, Radice S, Arnozis PA and Caso OH (1999). In vitro recurrent selection of potato; production and characterization of salt tolerant cell lines and plants. Plant Cell Tiss Organ Cult., 55: 1-8.

Peethambaran PK, Glenz R, Höninger S, Islam SMS, Hummel S, Harter K, Kolukisaoglu U, Meynard D, Guiderdoni E, Nick P and Riemann M (2018). Salt-inducible expression of OsJAZ8 improves resilience against salt-stress. BMC Plant Biology, 18: 311.

Rahman MH, Islam R, Hossain M and Haider SA (2008). Differential response of potato under sodium chloride stress conditions in vitro. Journal of Bio-Sci., 16: 79-83.

Rahnama $\mathrm{H}$ and Ebrahimzadeh $\mathrm{H}$ (2005). The effect of $\mathrm{NaCl}$ on antioxidant enzyme activities in potato seedlings. Biologia Plantarum. 49: 93-97.

Ranalli P, Di-Candilo M, Ruaro G and Marino A (1996). Drought effects on chlorophyll fluorescence and canopy temperature. 14th Triennial Conf. of the European Association for Potato Research, 2-7 May 1996, Sorrento, Italy.

Rengasamy P (2006). World salinization with emphasis on Australia. J Exp. Bot., 57: 1017-1023.

Scott GJ (2011). Plants, people, and the conservation of biodiversity of potatoes in Peru. Natureza Conservação. 9: 21-38.

Shahzad A, Ahmad M, Iqbal M, Ahmed I and Ali GM (2012). Evaluation of wheat land race genotypes for salinity tolerance at vegetative stage by using morphological and molecular markers. Genet. Mol. Res., 11(1): 679-692.

Siddique AB, Ara I, Islam SMS and Tuteja N (2014) Effect of air desiccation and salt stress factors on in vitro regeneration of rice (Oryza sativa L.). Plant Signaling Behavior, 9: 12.

Tuteja N and Sopory SK (2008). Chemical signaling under abiotic stress environment in plants. Plant Signaling Behavior, 3: 525-36.

Wang WB, Vinocur A and Altman (2003). Plant responses to drought, salinity and extreme temperatures: towards genetic engineering for stress tolerance. Planta, 218: 1-14.

Ward JH (1963). Hierarchical grouping to optimize an objective function. J Am Stat Assoc., 58(301): 236-244.

Yadav DK, Islam SMS and Tuteja N (2012) Rice heterotrimeric G-protein Gamma subunits (RGG1 and RGG2) are differentially regulated under abiotic stress. Plant Signaling and Behavior, 7: 7.

Yokoi S, Bressan RA and Hasegawa PM (2002). Salt stress tolerance of plants. JIRCAS Working Report, pp. 25-33.

Zhang Y and Donnelly D (1997). In vitro bioassay for salinity tolerance screening of potato. Potato Res., 40: 285-295.

(Manuscript received on June 23, 2019 and revised on August 29, 2019) 